\title{
Avaliação do local de nascimento de recém-natos com idade gestacional inferior a 34 semanas segundo complexidade da Unidade Neonatal em maternidades vinculadas à Rede Cegonha: Brasil, 2016-2017
}

\author{
Evaluation of the birthplace of newborns with gestational age less \\ than 34 weeks according to the complexity of the Neonatal Unit \\ in maternity hospitals linked to the "Rede Cegonha": \\ Brazil, 2016-2017
}

\author{
Barbara Vasques da Silva Ayres (https://orcid.org/0000-0002-6228-5932) ${ }^{1}$ \\ Rosa Maria Soares Madeira Domingues (https://orcid.org/0000-0001-5722-8127) ${ }^{2}$ \\ Marcia Leonardi Baldisserotto (https://orcid.org/0000-0001-6907-2510) ${ }^{1}$ \\ Neide Pires Leal (https://orcid.org/0000-0001-8793-1112) ${ }^{1}$ \\ Fernando Lamy-Filho (https://orcid.org/0000-0002-7858-4195) ${ }^{3}$ \\ Ana Paula da Cruz Caramachi (https://orcid.org/0000-0002-7968-3427) ${ }^{4}$ \\ Natali Pimentel Minoia (https://orcid.org/0000-0002-3505-0572) ${ }^{4}$ \\ Elaine Fernandes Viellas (https://orcid.org/0000-0001-5259-8102) ${ }^{1}$
}

${ }^{1}$ Escola Nacional de Saúde Pública, Fiocruz. R. Leopoldo Bulhões 1480, Manguinhos. 21041-210 Rio de Janeiro RJ Brasil. barbara.vsayres@gmail.com ${ }^{2}$ Instituto Nacional de Infectologia Evandro Chagas, Fiocruz. Rio de Janeiro RJ Brasil.

${ }^{3}$ Departamento de Medicina III, Universidade Federal do Maranhão. São Luís MA Brasil.

${ }^{4}$ Ministério da Saúde.

Brasília DF Brasil.

\begin{abstract}
This study aims to evaluate the birthplace of preterm infants with less than 34 gestational weeks at birth by type of neonatal care service in maternity hospitals of the "Rede Cegonha" and estimate the maternal factors associated with the inadequate place of birth for gestational age. This national cross-sectional study was performed in 2016/2017 to evaluate health establishments with the Rede Cegonha's action plan. Information was analyzed from 303 puerperae and the respective health establishments of their births. Newborns were classified by gestational age at birth ( $<30$ and 30-33 weeks) and health establishments as hospitals with neonatal intensive care service, hospitals with intermediate neonatal care service, and hospitals without neonatal care service. Ministerial Ordinance $N^{\circ}$ 930/2012 was used to classify the birthplace as appropriate for the newborn's gestational age. Preterm birth prevalence was 37.3 at less than 30 weeks' gestation and 66.8 at 30-33 weeks. Birth in inappropriate services for the newborn's gestational age occurred in $6.3 \%$, with significant regional and social differences. Inequalities in access to neonatal care for preterm infants persist in the "Rede Cegonha" despite advances.

Key words Preterm newborn, Neonatal intensive care unit, Maternal and child health, Maternal and child health services, Health policy
\end{abstract}

Resumo O objetivo deste estudo é avaliar o local de nascimento dos prematuros com menos de 34 semanas gestacionais segundo tipo de serviço de assistência neonatal em maternidades da Rede Cegonha, bem como estimar os fatores maternos associados ao local de nascimento não apropriado para idade gestacional. Estudo seccional, de âmbito nacional, realizado em 2016/2017, para avaliação de estabelecimentos com plano de ação da Rede Cegonha. Foram analisadas informações de 303 puérperas e os respectivos locais de ocorrência do parto. Os recém-natos foram classificados segundo a idade gestacional ao nascer $(<30$ e 30 a 33 semanas) e os serviços de saúde como unidade de cuidados intensivos neonatais, cuidados intermediários, sem serviço de cuidados neonatais. A portaria ministerial $n^{\circ}$ 930/2012 foi utilizada para classificar o local de nascimento como apropriado para a idade gestacional do recém-nato. A prevalência de nascimento pré-termo com menos de 30 semanas gestacional foi 37,3 e entre 30 e 33 semanas de 66,8. O nascimento em serviços não apropriado para a idade gestacional do recémnascido ocorreu em 6,3\%, com importantes diferenças regionais e sociais. Apesar dos avanços ainda persistem inequidades no acesso aos cuidados neonatais de prematuros na Rede Cegonha.

Palavras-chave Recém-nascido prematuro, Unidade de terapia intensiva neonatal, Saúde materno-infantil, Serviços de saúde materno-infantil, Política de saúde 


\section{Introdução}

O nascimento pré-termo é um importante problema de saúde coletiva, pois é a causa principal de mortalidade infantil e a segunda entre crianças até 5 anos $^{1,2}$. Somado a isso, está associado a diversos problemas de saúde na criança a médio e longo prazo ${ }^{3}$.

Anualmente, cerca de 30 milhões de recémnascidos apresentam risco de vida, sendo que dois terços destes são prematuros ${ }^{4}$. Estima-se que 14,8 milhões de nascimentos no mundo são prematuros, o que representa $11 \%$ de todos os nascidos vivos. $\mathrm{O}$ nascimento pré-termo afeta tanto países de alta como de baixa renda, sendo a China o país com maior número absoluto e os Estados Unidos o quarto ${ }^{5}$.

Organização Mundial da Saúde (OMS) define o nascimento pré-termo como os que ocorrem entre a $20^{\mathrm{a}}$ e $37^{\mathrm{a}}$ semana de gestação ${ }^{6}$. Este pode ser classificado de acordo com o período da semana gestacional em pré-termo extremo $(<28$ semanas), muito pré-termo ( $28 \mathrm{a}<32$ semanas $)$ e pré-termo moderado ( $32 \mathrm{a}<37$ semanas). Sendo que, dentro dessa última categoria, os nascimentos ocorridos da $34^{\mathrm{a}}$ e $36^{\mathrm{a}}$ semana gestacional são denominados de pré-termo tardio ${ }^{4}$.

No Brasil, o Inquérito Nacional sobre Partos e Nascimentos realizado em 2011-2012 estimou uma taxa de nascimento pré-termo de $11,5 \%$ (IC 95\% $10.3 \%-12.9 \%$ ), sendo $74 \%$ classificados como tardios, sem variações significativas segundo macrorregião do país e tipo de financiamento do parto (público ou privado) ${ }^{7}$. Recém-nascidos prematuros, em comparação aos nascidos a termo, apresentam limitações importantes para responderem aos estímulos extrauterinos e reagirem imunologicamente à presença dos microrganismos, exigindo cuidados extensos, integrais, que se iniciam no período pós natal imediato e podem se estender durante toda a infância ${ }^{8,9}$.

Uma rede assistencial à saúde materna infantil integrada, hierarquizada e regionalizada baseada na classificação de risco materno permitirá que as parturientes e seus bebês recebam os cuidados necessários, resultando em maior sobrevida de ambos. Para isto, as unidades de saúde necessitam estar equipadas para lidar com as complicações, estando munidas de infraestrutura física e equipe capacitada ${ }^{10,11}$.

No Brasil, a portaria $\mathrm{n}^{\circ} 930$ de 10 de maio de $2012^{12}$ recomenda que recém-natos (RN) com idade gestacional inferior a 30 semanas devam ser internados em Unidades de Cuidados Inten- sivos Neonatais $\left(\right.$ UTIN) ${ }^{12}$. Não existe recomendação de admissão em unidades neonatais do tipo Unidade de Cuidado Intermediário Neonatal Convencional (UCINCo) ou Unidade de Cuidado Intermediário Neonatal Canguru (UCINCa) baseada apenas na idade gestacional do RN. Entretanto, recém-natos com idade gestacional inferior a 34 semanas são considerados de alto risco, exigindo uma atenção diferenciada ${ }^{13}$.

O objetivo deste estudo é avaliar o local de nascimento do RN com idade gestacional inferior a 34 semanas segundo o tipo de serviço de assistência neonatal apropriado para sua idade gestacional, em maternidades vinculadas à Rede Cegonha, bem como verificar os fatores associados a nascimentos ocorridos em locais não apropriados e a ocorrência de desfechos precoces nesse local.

\section{Métodos}

Este estudo é parte da "Avaliação de práticas de cuidado ao parto e nascimento em maternidades da Rede Cegonha" desenvolvida pela Universidade Federal do Maranhão (UFMA) e pela Escola Nacional de Saúde Pública Prof. Sérgio Arouca (ENSP) da Fundação Oswaldo Cruz (Fiocruz), no período 2016-2017, sob a coordenação do Ministério da Saúde.

Esta avaliação teve como objetivo verificar as boas práticas na atenção ao parto e nascimento em todos os hospitais do Brasil com o Plano de Ação Regional na Rede Cegonha (RC), públicos ou privados conveniados ao SUS, com pelo menos 500 partos/ano em 2015, totalizando 606 estabelecimentos de saúde. $\mathrm{O}$ volume de partos nesses hospitais representava quase $50 \%$ dos partos ocorridos naquele ano no país, segundo o Sistema de Informações sobre Nascidos Vivos SINASC $^{14}$.

Todas as mulheres que tiveram parto hospitalar no período do estudo foram consideradas elegíveis para o estudo principal, sendo excluídas apenas aquelas com transtorno mental grave, que não compreendiam o idioma português, surdas ou internadas na Unidade Intermediária ou Unidade de Terapia Intensiva no pós-parto. Todas as puérperas elegíveis internadas em cada unidade hospitalar no período de duração do trabalho de campo foram convidadas a participar do estudo. Esse período foi definido pela macrorregião na qual o hospital estava localizado, sendo de três dias nas regiões Sudeste e Nordeste, seis dias na região Norte e Sul e oito dias na região Centro-Oeste. 
Foram entrevistadas 10.665 puérperas, 10.555 que tiveram nascidos vivos, independentemente do peso e da idade gestacional, 83 natimortos com peso maior que 500 gramas e/ou idade gestacional maior que 22 semanas.

Os métodos de coleta de dados utilizados foram: entrevistas face a face com informanteschaves (puérperas, profissionais de saúde e gestores), extração de dados de prontuário, análise de documentos e observação in loco. Informações mais detalhadas da metodologia do estudo estão disponíveis em Vilella et al. ${ }^{15}$.

Para o presente estudo, foram utilizadas as informações obtidas na entrevista com as mulheres, na extração de dados de prontuário e na observação in loco. As entrevistas com as mulheres foram realizadas durante a internação hospitalar, no mínimo 6 horas após o parto. Um instrumento eletrônico foi desenvolvido especificamente para este estudo, contendo perguntas sobre características sociodemográficas, informações sobre acesso ao hospital/maternidade, acolhimento e atenção hospitalar durante a assistência ao trabalho de parto e parto, dentre outras. Dados do prontuário hospitalar foram obtidos logo após a entrevista com a puérpera, através de um instrumento para extração de dados contendo informação da assistência prestada à mulher e ao recémnascido, bem como alguns desfechos neonatais ocorridos até aquele momento da internação. A observação in loco buscou avaliar os processos de cuidado, condições de infraestrutura e planta física. Um roteiro previamente elaborado serviu de guia para o avaliador enquanto o mesmo percorria o caminho feito pelas puérperas - porta de entrada, recepção, admissão, setor de observação, enfermaria para gestação de alto risco, ambiente pré-parto, parto, alojamento conjunto e Unidade Neonatal.

Para as análises deste estudo, foram incluídas as mulheres com partos de nascidos vivos com idade gestacional inferior a 34 semanas, sendo excluídas aquelas que tiveram como resultado da gestação feto natimorto ou nascido vivo com idade gestacional ignorada (43).

Para o cálculo da idade gestacional, foram utilizados dados do prontuário hospitalar, sendo utilizado um algoritmo já adotado previamente, que privilegia o cálculo da idade gestacional a partir de informações provenientes de exames de ultrassonografia realizadas durante o pré-natal ${ }^{16}$.

$\mathrm{Na}$ primeira etapa da análise, as características sociodemográficas e obstétricas das mulheres foram descritas segundo idade gestacional do RN ( $<30$ semanas e de 30-33 semanas). Foram ava- liadas: região de residência (Norte, Nordeste, Sul, Sudeste, Centro-Oeste); cor da pele autorreferida (branca, preta, parda, amarela, indígena); escolaridade (menos que ensino fundamental, ensino fundamental completo, ensino médio e mais); situação conjugal (com ou sem companheiro); paridade (primípara, 1 a 2 partos anteriores, 3 ou mais partos anteriores); gravidez planejada ( sim ou não); condição do RN no momento da entrevista (vivo ou óbito neonatal); e peregrinação (busca de mais de um serviço hospitalar para a internação para a assistência ao parto, sim ou não).

A seguir, a proporção de recém-natos que nasceu em local não apropriado, segundo sua idade gestacional, bem como sua distribuição, segundo características maternas, foi estimada. A portaria ministerial no 930/2012 foi a referência normativa utilizada para classificação do local de nascimento como apropriado para a idade gestacional: local apropriado $=$ se idade gestacional $<30$ semanas e admissão em serviço com leitos de UTIN; se idade gestacional 30-33 semanas e admissão em serviço com leitos de UTIN ou UCINCo/UCINCa; local não apropriado = se idade gestacional $<30$ semanas e admissão em serviço sem leitos de UTIN; se idade gestacional 30-33 semanas e admissão em serviços sem leitos de UTIN/UCINCo/UCINCa.

Para estimar a associação entre local de nascimento não apropriado para a idade gestacional e as características sociodemográficas e obstétricas maternas foram realizados modelos de regressões logísticos simples e múltiplo. Devido ao pequeno número de nascimentos em cada faixa gestacional, foi considerada como variável desfecho o local não apropriado de nascimentos dos RN com menos de 34 semanas gestacionais. Como variáveis explicativas foram utilizadas características maternas que poderiam estar associadas às dificuldades de acesso a serviços neonatais de maior complexidade. Foram incluídas no modelo de regressão múltiplo, todas as variáveis que apresentaram um p-valor associado ao coeficiente de regressão inferior a 0,20 na regressão logística simples. Mulheres que se autodeclararam amarelas ou indígenas foram excluídas desta etapa da análise devido ao pequeno número de casos (1,6\% e $0,7 \%$, respectivamente). Dessas análises, foram obtidas Razões de Chances (RC) brutas e ajustadas, assim como seus intervalos de confiança (IC) de 95\%.

Como última etapa, foi realizada uma descrição dos desfechos precoces dos recém-natos prematuros, incluindo o local de internação, transfe- 
rências e ocorrência de óbito neonatal ocorridos até o momento da entrevista materna.

Em todas as análises, foram utilizados procedimentos para amostras complexas, com utilização de ponderação pelo inverso da probabilidade da inclusão de cada puérpera, calibração para garantir que a distribuição das puérperas amostradas correspondesse à distribuição de partos ocorridos nos 606 hospitais no ano de 2017, e utilização do efeito de desenho. Todas as análises foram realizadas no software SPSS versão 22.

O estudo de avaliação foi aprovado pelo Comitê de Ética em Pesquisa com Seres Humanos da Universidade Federal do Maranhão e da Escola Nacional de Saúde Pública Sérgio Arouca, em 14 de dezembro de 2016. Antes da entrevista, a todas as mulheres e gestores foi lido o Termo de Consentimento Livre e Esclarecido (TCLE), sendo entregue uma cópia àqueles que concordaram em participar da avaliação. Todos os cuidados foram adotados visando garantir o sigilo e a confidencialidade das informações.

\section{Resultados}

Dos 10.539 nascidos vivos, 303 (2,9\% IC 95\%: 2,4\%-3,4\%) apresentavam idade gestacional inferior a 34 semanas ao nascer, sendo $33,2 \%$ com IG inferior a 30 semanas e $66,8 \%$ com IG entre 30 e 33 semanas. A maior parte das mulheres residia nas regiões Nordeste $(37,3 \%)$ e Sudeste $(29,6 \%)$ do país, se declarou de cor de pele parda $(59,3 \%)$ e apresentava ensino médio ou mais $(43,8 \%)$. Um quinto das mulheres era adolescente $(20,8 \%)$, $23,6 \%$ não possuíam companheiro e $43,3 \%$ estavam tendo seu primeiro parto. A gestação não foi planejada pela maioria das mulheres $(60,2 \%)$, e $37,9 \%$ relataram ter buscado mais de um serviço para admissão para o parto. Não foram verificadas diferenças significativas nas características maternas segundo IG ao nascimento (Tabela 1).

Do total de recém-nascidos com IG inferior a 34 semanas, $6,3 \%$ (IC $95 \% 3,7 \%-10,5 \%$ ) foram internados em hospitais com serviço de neonatologia não apropriado para a idade gestacional ao nascimento. Mulheres residentes na macrorregião centro-oeste $(10 \%)$, na faixa etária entre 30 a 34 anos $(8,6 \%)$, de cor de pele preta $(8,7 \%)$, com escolaridade menor que o ensino fundamental (12,1\%), com 3 ou mais partos anteriores $(14,2 \%)$, sem companheiro $(9,7 \%)$ e com gravidez não planejada $(8,7 \%)$ apresentaram maiores prevalências de local de nascimentos inapropriados (Tabela 2).
Na regressão logística não ajustada, a maior chance de nascimento em local não apropriado foi observada em mulheres com menor escolaridade ( $\mathrm{RC}=4,97$ - IC $=1,25-19,76)$ e maior paridade $(\mathrm{RC}=5,68$ - IC $=1,42-22,69$ ), sendo observado um gradiente nas associações, ou seja, quanto menor a escolaridade e quanto maior o número de partos, maior a chance de nascimento em serviços não apropriados para a idade gestacional. Com o ajuste das associações, o gradiente para a escolaridade foi mantido, mas com perda da significância estatística. A chance de nascimento em local não apropriado em mulheres com 3 ou mais partos anteriores foi aumentada após ajuste para as demais variáveis $(\mathrm{RC}=8,28$ - IC 95\% 1,34-51,11). Mulheres com 35 anos ou mais apresentaram menor chance do parto prematuro ocorrer em local não apropriado ( $\mathrm{RC}=$ 0,13 - IC $=0,17-0,95$ ) (Tabela 3).

A Figura 1 apresenta os desfechos precoces dos recém-natos até o momento das entrevistas com as mulheres no pós-parto, segundo as faixas de idade gestacional menor de 30 semanas e de 30 a 33 semanas. Dos recém-natos com menos de 30 semanas gestacionais, nove nasceram em maternidades sem UTI neonatal. Desses, um evoluiu para óbito precocemente e oito apresentaram registro de internação, sendo dois transferidos para outros serviços de maior complexidade e seis internados em condições adaptadas, dos quais dois evoluíram para óbito. Sendo assim, a mortalidade em recém-natos com IG inferior a 30 semanas internados em serviços não adequados ao seu risco foi de $33,3 \%$, enquanto naqueles internados em serviços adequados foi de 5,9\%. Dos recémnatos com idade gestacional de 30 a 34 semanas, 10 nasceram em serviços sem UCINCo/UCINCa, tendo apenas um deles o registro de internação feita em condições adaptadas, e os nove restantes provavelmente permanecidos em alojamento conjunto.

\section{Discussão}

No Brasil (DataSus) e no mundo, $85 \%$ dos nascimentos prematuros são moderados a tardios, ocorrendo na faixa gestacional de 32 a 36 semanas ${ }^{17,18}$. Os dados deste estudo, realizado em serviços de referência da Rede Cegonha, evidenciaram uma taxa de nascimento pré-termo com IG inferior a 34 semanas de 2,9\%. Esse resultado é similar ao encontrado em inquérito nacional realizado nos anos 2011-2012, no qual essa proporção foi de 3,0\%, correspondendo a $74 \%$ dos nas- 
Tabela 1. Características demográficas, sociais e obstétricas das mulheres segundo a idade gestacional ao nascimento. Rede Cegonha. Brasil, 2016-2017.

\begin{tabular}{|c|c|c|c|c|c|c|}
\hline \multirow[b]{2}{*}{ Características maternas } & \multirow[b]{2}{*}{$\mathbf{N}$} & \multirow[b]{2}{*}{$\%$} & \multicolumn{2}{|c|}{$\begin{array}{c}\text { Menos de } 30 \text { semanas } \\
\quad(n=101)\end{array}$} & \multicolumn{2}{|c|}{$\begin{array}{c}30 \text { a } 33 \text { semanas } \\
(n=202)\end{array}$} \\
\hline & & & $\%$ & IC & $\%$ & IC \\
\hline \multicolumn{7}{|l|}{ Regiões } \\
\hline Norte & 41 & 13,4 & 14,4 & $(7-27,5)$ & 12,8 & $(7,3-21,6)$ \\
\hline Nordeste & 113 & 37,3 & 32,4 & $(20,2-47,6)$ & 39,7 & $(29,5-50,9)$ \\
\hline Centro Oeste & 30 & 9,8 & 10,7 & $(5,3-20,4)$ & 9,4 & $(5,5-15,7)$ \\
\hline Sudeste & 90 & 29,6 & 30,5 & $(19,5-44,4)$ & 29,1 & $(20,5-39,5)$ \\
\hline Sul & 30 & 9,9 & 11,9 & $(6,7-20,4)$ & 8,9 & $(5,3-14,5)$ \\
\hline \multicolumn{7}{|l|}{ Idade } \\
\hline Menos de 20 anos & 63 & 20,8 & 22,4 & $(14,5-33)$ & 19,9 & $(14,5-26,7)$ \\
\hline 20 a 34 anos & 196 & 64,9 & 67,6 & $(56,9-76,8)$ & 63,6 & $(56,1-70,5)$ \\
\hline 35 anos ou mais & 43 & 14,3 & 9,9 & $(5,1-18,4)$ & 16,5 & $(10,7-24,5)$ \\
\hline \multicolumn{7}{|l|}{ Raça } \\
\hline Branca & 66 & 22,3 & 17,9 & $(9,7-30,6)$ & 24,5 & $(18,3-32,0)$ \\
\hline Preta & 47 & 15,6 & 19,5 & $(11,1-32,1$ & 13,6 & $(9-20)$ \\
\hline Parda & 176 & 59,3 & 60,1 & $(47,1-71,8)$ & 58,9 & $(50,8-66,6)$ \\
\hline Amarela/Oriental & 6 & 2,2 & 1,4 & $(0,3-5,7)$ & 2,5 & $(1-6,3)$ \\
\hline Indígena & 2 & 0,7 & 1,1 & $(0,2-7,6)$ & 0,5 & $(0,1-1,9)$ \\
\hline \multicolumn{7}{|l|}{ Escolaridade } \\
\hline Menos que fundamental & 90 & 30,0 & 37,1 & $(27,6-47,8)$ & 26,4 & $(20,6-33,2)$ \\
\hline Fundamental completo & 79 & 26,2 & 24,2 & $(16,1-34,6)$ & 27,2 & $(20,3-35,3)$ \\
\hline Médio completo ou mais & 133 & 43,8 & 38,7 & $(28,1-50,5)$ & 46,4 & $(38,6-54,4)$ \\
\hline \multicolumn{7}{|l|}{ Possui companheiro } \\
\hline Não & 71 & 23,6 & 27,3 & $(18,1-39)$ & 21,7 & $(15,9-29)$ \\
\hline Sim & 231 & 76,4 & 72,7 & $(61-81,9)$ & 78,3 & $(71-84,1)$ \\
\hline \multicolumn{7}{|l|}{ Paridade } \\
\hline Primipara & 129 & 43,3 & 41,6 & $(30,8-53,3)$ & 44,1 & $(36,3-52,2)$ \\
\hline 1 a 2 partos anteriores & 126 & 42,5 & 44,5 & $(32,7-56,8)$ & 41,5 & $(33,6-49,8)$ \\
\hline 3 ou mais partos anteriores & 43 & 14,3 & 14 & $(7,5-24,4)$ & 14,4 & $(9,8-20,8)$ \\
\hline Gravidez planejada & & & - & - & & \\
\hline Não & 182 & 60,2 & 60,7 & $(49,3-71)$ & 60 & $(51,8-67,7)$ \\
\hline Sim & 121 & 39,8 & 39,3 & $(29-50,7)$ & 40 & $(32,3-48,2)$ \\
\hline \multicolumn{7}{|l|}{ Peregrinação } \\
\hline Não & 187 & 62,1 & 62 & $(49,1-73,5)$ & 62,4 & $(53,3-70,7)$ \\
\hline Sim & 114 & 37,9 & 38 & $(26,5-50,9)$ & 37,6 & $(29,3-46,7)$ \\
\hline
\end{tabular}

cimentos prematuros ${ }^{19}$. Costa et al. ${ }^{20}$, ao avaliar informações do SINASC para o ano de competência de 2013, encontraram 1,0\% dos nascimentos do SUS dentro da faixa de idade gestacional inferior a 32 semanas.

Diversos fatores maternos (características sociodemográficas, obstétricas, psicológicas e genéticas), ambientais e paternos têm sido associados a nascimentos prematuros ${ }^{18,21}$. Neste estudo, não foram comparadas características maternas de recém-natos prematuros e a termo. No entanto, a distribuição dos nascimentos investigados mostrou uma maior proporção de prematuros nas regiões Centro-Oeste, Norte e Nordeste, as menos desenvolvidas economicamente, e menor proporção nas regiões Sul e Sudeste.

A proporção de mães adolescentes foi também maior entre os prematuros $(20,8 \% \text { vs } 16,5 \%)^{22}$, de forma semelhante a outros estudos que apontam maior prevalência de nascimento pré-termo em mulheres dos extremos etários ${ }^{23,24}$. O nível de escolaridade também diferiu do observado para o total de nascimentos pré-termo no Brasil, com proporções mais elevadas entre as mulheres com 
Tabela 2. Características demográficas, sociais e obstétricas das mães de recém-natos com idade gestacional inferior a 34 semanas gestacionais segundo classificação do local de nascimentos. Rede Cegonha Brasil, 2016-2017.

\begin{tabular}{|c|c|c|c|c|}
\hline \multirow{3}{*}{$\begin{array}{c}\text { Características } \\
\text { maternas }\end{array}$} & \multicolumn{4}{|c|}{ Local de nascimento } \\
\hline & \multicolumn{2}{|c|}{$\begin{array}{c}\text { Não } \\
\text { apropriado } \\
\text { para IG } \\
\end{array}$} & \multicolumn{2}{|c|}{$\begin{array}{c}\text { Apropriado } \\
\text { para IG }\end{array}$} \\
\hline & $\mathbf{N}$ & $\%$ & $\mathbf{N}$ & $\%$ \\
\hline \multicolumn{5}{|l|}{ Regiões } \\
\hline Norte & 3 & 7,3 & 38 & 92,7 \\
\hline Nordeste & 9 & 7,9 & 104 & 92,1 \\
\hline Centro Oeste & 3 & 10,0 & 27 & 90,0 \\
\hline Sudeste & 4 & 4,5 & 85 & 95,5 \\
\hline Sul & 1 & 3,3 & 29 & 96,7 \\
\hline \multicolumn{5}{|l|}{ Idade } \\
\hline Menos de 20 anos & 5 & 7,9 & 58 & 92,1 \\
\hline 20 a 29 anos & 9 & 6,5 & 130 & 93,5 \\
\hline 30 a 34 anos & 5 & 8,6 & 53 & 91,4 \\
\hline 35 anos ou mais & 1 & 2,3 & 43 & 97,7 \\
\hline \multicolumn{5}{|l|}{ Raça } \\
\hline Branca & 3 & 4,5 & 63 & 95,5 \\
\hline Preta & 4 & 8,7 & 42 & 91,3 \\
\hline Parda & 10 & 5,7 & 166 & 94,3 \\
\hline Amarela/Oriental & 0 & 0 & 6 & 100 \\
\hline Indígena & 0 & 0 & 2 & 100 \\
\hline \multicolumn{5}{|l|}{ Escolaridade } \\
\hline $\begin{array}{l}\text { Menos que } \\
\text { fundamental }\end{array}$ & 11 & 12,1 & 80 & 87,9 \\
\hline $\begin{array}{l}\text { Fundamental } \\
\text { completo }\end{array}$ & 5 & 6,3 & 74 & 93,7 \\
\hline $\begin{array}{l}\text { Médio completo ou } \\
\text { mais }\end{array}$ & 3 & 2,3 & 129 & 97,7 \\
\hline \multicolumn{5}{|l|}{ Paridade } \\
\hline Primípara & 4 & 3,1 & 125 & 96,9 \\
\hline 1 a 2 partos anteriores & 7 & 5,5 & 119 & 94,5 \\
\hline $\begin{array}{l}3 \text { ou mais partos } \\
\text { anteriores }\end{array}$ & 6 & 14,2 & 36 & 85,8 \\
\hline \multicolumn{5}{|l|}{ Possui companheiro } \\
\hline Não & 7 & 9,7 & 65 & 90,3 \\
\hline Sim & 12 & 5,2 & 219 & 94,8 \\
\hline \multicolumn{5}{|l|}{ Gravidez planejada } \\
\hline Não & 15 & 8,7 & 167 & 91,3 \\
\hline Sim & 3 & 2,5 & 117 & 97,5 \\
\hline \multicolumn{5}{|l|}{ Peregrinação } \\
\hline Não & 16 & 3,2 & 171 & 96,8 \\
\hline Sim & 3 & 2,6 & 110 & 97,4 \\
\hline Total & 19 & 6,3 & 284 & 93,7 \\
\hline
\end{tabular}

escolaridade inferior ao ensino fundamental ou com ensino médio. A menor escolaridade, fator relacionado à maior vulnerabilidade social, está associada aos nascimentos prematuros espontâneos ${ }^{7}$. A maior proporção de mulheres com ensino médio completo ou mais pode ser decorrente de um efeito de coorte geracional, já que a proporção de gestantes em idade avançada foi elevada e essas, geralmente, apresentam maior escolaridade do que as adolescentes. Em relação à gravidez na adolescência, recente revisão integrativa evidenciou a influência do pré-natal e do nível de escolaridade no desfecho prematuridade, concluindo que os riscos obstétricos e fetais podem ser minimizados se houver uma rede de apoio direcionada às adolescentes, que foquem na atenção reprodutiva, pré-natal e suporte social $^{25}$. Maior prevalência foi também observada em mulheres de cor não branca, principalmente as pardas e pretas, população de maior vulnerabilidade social no contexto brasileiro ${ }^{26}$.

A proporção de mulheres que referiram ter procurado mais de um serviço para admissão para assistência ao parto $(37,9 \%)$ foi superior ao encontrado em inquérito nacional anterior, que estimou $16,2 \%$ de peregrinação $0^{27}$. A peregrinação, ao retardar o acesso à assistência hospitalar e aumentar a demora para o recebimento de uma assistência adequada, tem sido associada a desfechos maternos ${ }^{27,28}$ e neonatais ${ }^{29,30}$ negativos. Uma possível explicação para esse resultado é o fato das maternidades da Rede Cegonha serem, em geral, maternidades de referência em seus municípios/estados, fazendo com que as mulheres tenham sido internadas nesses serviços após terem buscado um primeiro atendimento em outro de menor complexidade.

$O$ resultado encontrado de que a peregrinação não se mostrou associada ao local de nascimento não apropriado aos recém-natos prematuros vai ao encontro da hipótese do presente estudo, refletindo a busca por serviços de maior complexidade existentes no âmbito da Rede Cegonha. Da mesma forma, o estudo de Menezes et al. ${ }^{31}$ não encontrou associação entre a peregrinação anteparto e o risco da gestante no momento da internação, o que pode indicar que o processo de peregrinação ocorre em busca de disponibilidade de vagas e não necessariamente em busca de recursos adequados às necessidades do risco gestacional.

Um indicador usado para avaliar a saúde perinatal na Europa é o percentual de nascimento de recém-nascidos muito prematuros em unidades sem UTI Neonatal ${ }^{32}$. Bélgica, França e Alemanha tiveram mais de $75 \%$ dos partos de nascido vivos com IG inferior a 32 semanas ocorridos em 
Tabela 3. Regressão logística e múltipla dos fatores associados ao local de nascimento inapropriado para recémnatos com idade gestacional inferior a 34. Rede Cegonha, Brasil, 2016-2017.

\begin{tabular}{|c|c|c|c|c|}
\hline \multirow[b]{2}{*}{ Características maternas } & \multicolumn{4}{|c|}{$\begin{array}{l}\text { Prematuro extremo nascido em unidade } \\
\text { não apropriada a idade gestacional }\end{array}$} \\
\hline & RC bruta & IC & RC ajustada & IC \\
\hline \multicolumn{5}{|l|}{ Região } \\
\hline Norte & 3,89 & $(0,37-40,95)$ & & \\
\hline Nordeste & 4,77 & $(0,51-44,96)$ & & \\
\hline Centro Oeste & 5,77 & $(0,55-60,58)$ & & \\
\hline Sudeste & 2,86 & $(0,27-30,22)$ & & \\
\hline Sul & 1 & & & \\
\hline \multicolumn{5}{|l|}{ Idade * } \\
\hline Menos de 20 anos & 1,05 & $(0,36-3,07)$ & 1,45 & $(0,39-5,34)$ \\
\hline 20 a 34 anos & 1 & & 1 & \\
\hline 35 anos ou mais & 0,24 & $(0,05-1,18)$ & 0,13 & $(0,17-0,95)$ \\
\hline \multicolumn{5}{|l|}{ Cor da pele } \\
\hline Branca & 1 & & & \\
\hline Preta & 1,74 & $(0,25-12,07)$ & & \\
\hline Parda & 1,13 & $(0,30-4,31)$ & & \\
\hline \multicolumn{5}{|l|}{ Escolaridade ${ }^{\star}$} \\
\hline Menos que fundamental & 4,97 & $(1,25-19,76)$ & 1,95 & $(0,37-10,20)$ \\
\hline Fundamental completo & 2,61 & $(0,49-14,00)$ & 1,48 & $(0,33-6,70)$ \\
\hline Médio completo ou mais & 1 & & 1 & \\
\hline \multicolumn{5}{|l|}{ Paridade * } \\
\hline Primípara & 1 & & 1 & \\
\hline 1 a 2 partos anteriores & 2,08 & $(0,64-6,73)$ & 1,88 & $(0,54-6,51)$ \\
\hline 3 ou mais partos anteriores & 5,68 & $(1,42-22,69)$ & 8,28 & $(1,34-51,11)$ \\
\hline \multicolumn{5}{|l|}{ Vive com companheiro } \\
\hline Não & 1,76 & $(0,60-5,20)$ & & \\
\hline Sim & 1 & & & \\
\hline \multicolumn{5}{|l|}{ Gravidez planejada * } \\
\hline Não & 3,14 & $(0,91-10,86)$ & 2,59 & $(0,60-11,20)$ \\
\hline Sim & 1 & & 1 & \\
\hline \multicolumn{5}{|l|}{ Peregrinação } \\
\hline Não & 1 & & & \\
\hline Sim & 0,3 & $(0,05-1,95)$ & & \\
\hline
\end{tabular}

unidades com UTI Neonatal ${ }^{32,33}$. Estudo realizado com puérperas atendidas no SUS do Rio de Janeiro destaca que 29\% das puérperas de alto risco realizaram seu parto em estabelecimentos desprovidos de UTI Neonatal ${ }^{32}$. No presente estudo, 6,3\% dos recém-natos com menos de 34 semanas gestacionais nasceram em estabelecimentos com serviços de neonatologia não apropriados para sua idade gestacional. Como só foram incluídos serviços localizados em região de saúde com plano de ação da Rede Cegonha ${ }^{34}$, que é uma estratégia de organização da linha de cuidado à gestante e à criança até os dois anos de idade, deve-se supor que esse fato explique que os resultados encontrados sejam melhores do que os estudos realizados em hospitais não integrados à Rede Cegonha.

Um estudo que analisou internações em serviços com unidade de terapia neonatal, utilizando como marcadores de gravidade o peso ao nascer inferior a $1.500 \mathrm{~g}$ e o escore de Apgar no $5^{\circ}$ minuto inferior a 7 , encontrou $38 \%$ de nascimentos com marcador de gravidade ocorrendo em estabelecimentos sem terapia intensiva. Embora os critérios adotados sejam diferentes, alguns resultados, como a maior proporção de internação em local apropriado nas regiões Sul e Sudeste, são semelhantes ao deste estudo ${ }^{20}$. 

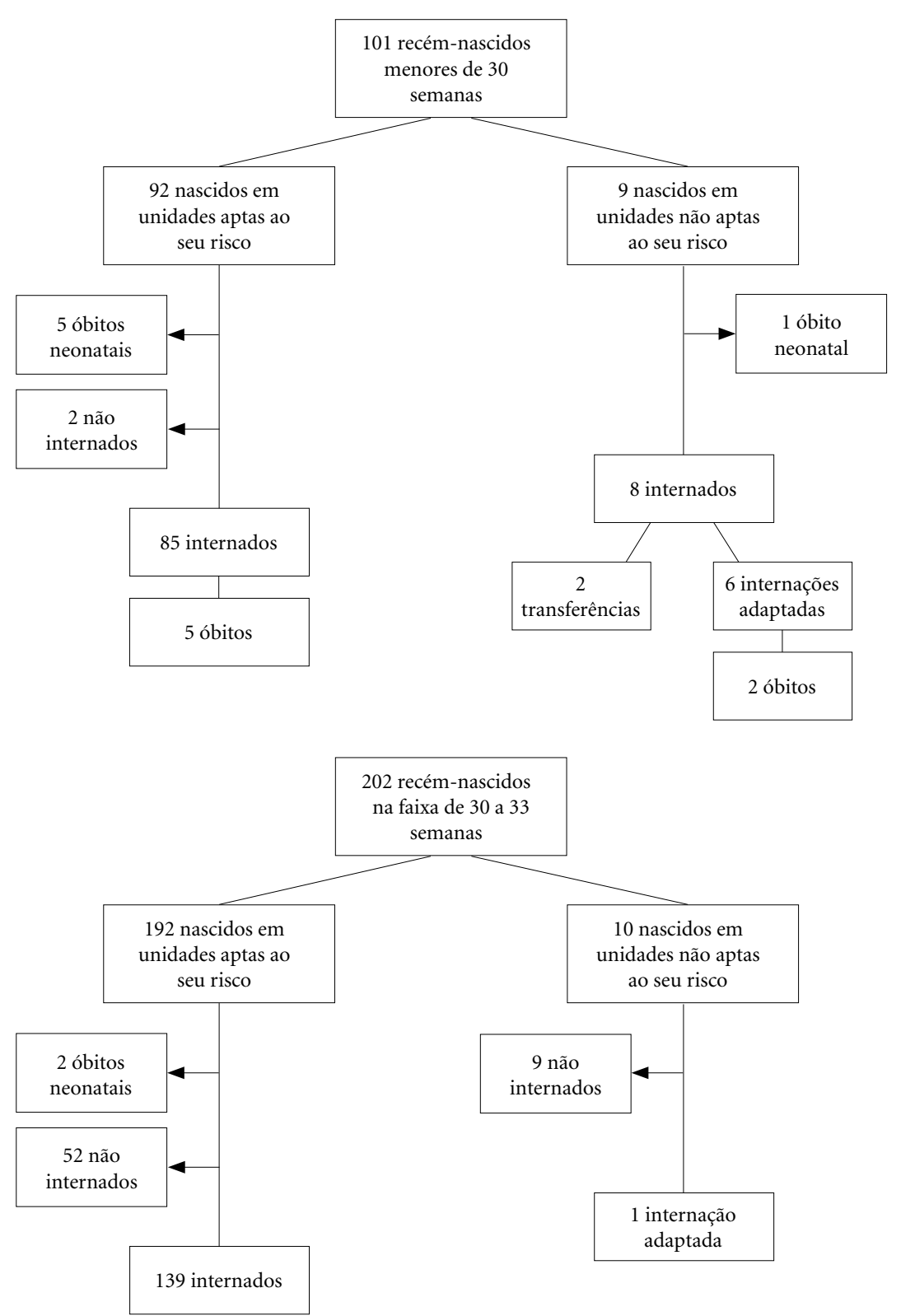

Figura 1. Desfechos precoces de recém-natos com menos de 34 semanas gestacionais. Rede Cegonha, Brasil, 2016-2017.

Em 2011-2012, apenas 39,3\% dos hospitais públicos dispunham de UTI neonatal, não estando disponíveis dados sobre UCINCo ${ }^{35}$. Nessa ocasião, as regiões Norte, Nordeste e Centro-Oeste apresentaram as menores proporções de serviços com UTIN, embora mostrassem prevalências semelhantes de nascimento pré-termo extremo ${ }^{35}$. Neste estudo, essas mesmas regiões apresentaram as proporções mais elevadas de internação em serviços não apropriados à idade gestacional. Embora essas diferenças não tenham sido significativas, provavelmente pelo pequeno tamanho amostral, podem estar indicando desigualdades na distribuição de leitos de cuidado neonatal. A sobrevida e a qualidade de vida são maiores para prematuros extremos que nascem em hospitais com serviço de cuidado intensivo neonatal. Na França, residir em local distante de serviços com 
UTIN reduziu a chance de internações em serviços especializados, mesmo quando ajustado para características maternas e obstétricas ${ }^{36}$.

Ressalte-se a observação de internações adaptadas de recém-natos prematuros com menos de 30 semanas gestacionais em serviços da Rede Cegonha que não dispunham de leitos de UTIN. Ainda que os números sejam pequenos, a taxa de mortalidade nessas internações adaptadas foi seis vezes maior do que a observada em hospitais com UTI neonatal (33,3\% vs 5,9\%). A ocorrência de óbitos nas internações não adaptadas reforça a ideia de que o fato do nascimento ocorrer em local provido de Unidade Neonatal apropriada para IG não é garantia para que haja acesso aos cuidados oportunos da mesma ${ }^{32}$.

A maior proporção de nascimentos em local não apropriado foi observada em mulheres de menor escolaridade, com gestações não planejadas e com maior número de partos, embora apenas a paridade tenha mantido associação significativa. A associação entre menor escolaridade e local não apropriado para o risco da puérpera foi descrito por Menezes ${ }^{32}$, no qual a chance era $30 \%$ maior de mulheres com baixa escolaridade terem seus filhos em estabelecimentos sem Unidade Neonatal. A baixa escolaridade, a maior paridade e a gestação não planejada também se encontram associadas à menor utilização de serviços de assistência pré-natal, com maior proporção de não realização do acompanhamento, início tardio do pré-natal e menor número de consultas ${ }^{37}$. Amplo estudo de âmbito nacional, mostrou ainda evidências da associação da baixa escolaridade com a maior peregrinação para o parto ${ }^{36}$. Mulheres com gestações não planejadas, bem como aquelas de menor condição econômica e com pior qualidade de atenção ao parto e ao recém-nato, também apresentam maior prevalência de depressão pós-parto ${ }^{38}$. Na França, Zeitlin et al. ${ }^{39}$ avaliaram os fatores associados ao nascimento de prematuros extremos em serviços sem cuidado intensivo neonatal. $\mathrm{O}$ estudo identificou que o maior risco de prematuridade muitas vezes ocorre em virtude da dificuldade de acesso das gestantes aos serviços especializados, agravando os desfechos perinatais $^{39}$.

Este estudo apresenta algumas limitações. A primeira delas refere-se ao uso exclusivo da idade gestacional para definição do tipo de serviço de assistência neonatal necessário. Recém-natos com mais de 30 semanas gestacionais podem ter necessitado de cuidados intensivos (UTIN), mas foram considerados como local apropriado quando nasciam em estabelecimentos com
UCINCO, o que pode ter superestimado os nossos achados de nascimento em local apropriado. Entretanto, essa limitação não afeta o objetivo do estudo que é avaliar se o local de nascimento do RN com menos de 34 semanas foi apropriado para a sua idade gestacional.

A forma utilizada para calcular a idade gestacional também é uma limitação. A utilização de um algoritmo específico para a estimativa da idade gestacional mais precisa é passivo de erros, o que pode explicar, por exemplo, a observação de recém-natos com menos de 30 semanas gestacionais sem internação em serviço de cuidado intensivo e com evolução favorável. Cabe ressaltar que a verificação da situação do RN foi realizada no momento da entrevista materna, com identificação de óbitos neonatais ocorridos até aquele momento. É possível que alguns RN tenham evoluído para óbito posteriormente durante a internação hospitalar, não tendo, portanto, uma evolução favorável.

Por fim, não foram avaliados itens relacionados à estrutura e aos processos assistenciais dos serviços de assistência neonatal existentes e, nesses casos, os recém-natos podem ter sido internados em locais apropriados segundo sua tipologia, mas sem condições adequadas de funcionamento.

Mesmo frente a essas limitações, este estudo traz importantes contribuições para a compreensão dos fluxos de atendimento e dos fatores associados ao local de assistência aos RN's prematuros dentre as maternidades conveniadas à Rede Cegonha.

\section{Conclusão}

A prevalência de nascimento pré-termo abaixo de 34 semanas gestacionais no país é elevada, com importantes diferenças regionais e sociais. A proporção dos prematuros internados em serviços de cuidado neonatal intensivo ou intermediário não apropriados para a idade gestacional foi baixa, mas apresentou associação com fatores que indicam maior vulnerabilidade social das parturientes. Fato esse que demonstra ainda haver inequidades no acesso aos cuidados intensivos e intermediários aos recém-nascidos prematuros no âmbito das maternidades vinculadas à Rede Cegonha.

Ressalta-se, portanto, a importância da identificação precoce desse grupo de mulheres, visando garantir uma linha de cuidado adequada em toda a gestação, parto e pós-parto, evitando o surgimento de desfechos negativos em saúde. Es- 
pecificamente no momento da admissão hospitalar para o parto, a idade gestacional permitiria a transferência da gestante para serviços com estrutura mais adequada para assistência a recémnatos prematuros.

Somado a isso, pesquisas a longo prazo que acompanhem os desfechos nos recém-natos internados em locais não apropriados para idade gestacional são necessárias, a fim de esclarecer possíveis desfechos no RN que necessitam de maior tempo para ocorrerem, tal como os óbi- tos. Estudos futuros devem ser realizados a fim de avaliar a adequação dos serviços neonatais, visando a garantia de que recém-natos sejam internados em locais apropriados à sua idade gestacional e com condições adequadas de funcionamento.

Para a gestão, os achados do presente estudo confirmam o quão urgente é o investimento no sistema de saúde e nas políticas públicas voltadas para a ampliação da qualidade da atenção materna e neonatal nas maternidades da Rede Cegonha.

\section{Colaboradores}

BVS Ayres, RMSM Domingues, MC Leal, ML Baldisserotto e EF Viellas realizaram a concepção, metodologia, análise e interpretação dos dados. NP Leal, F Lamy-Filho, NP Minóia e APC Caramaschi fizeram uma revisão crítica do artigo Todos os autores aprovaram a versão aqui submetida. 


\section{Referências}

1. França EB, Lansky S, Rego MAS, Malta DCF, França JS, Teixeira R, Porto D, Almeida MF, Souza MFM, Szwarcwald CL, Mooney MN, Mohsen AMNV. Principais causas da mortalidade na infância no Brasil, em 1990 e 2015: estimativas do estudo de Carga Global de Doença. Rev Bras Epidemiol 2017; 20(Supl. 1):46-60.

2. Belizán JM, Hofmeyr J, Buekens P, Salaria N. Preterm birth, an unresolved issue. Reprod Health 2013; 10(1):58.

3. Beck S, Wojdyla D, Say L, Betran A. P, Merialdi M, Requejo JH, Rubens C, Menon R, Van Look PF. The worldwide incidence of preterm birth: a systematic review of maternal mortality and morbidity. Bull World Health Organ 2010; 88(1):31-38.

4. World Health Organization (WHO). Born too soon: the global action report on preterm birth. Geneva: WHO; 2012.

5. World Health Organization (WHO). Survive and thrive: transforming care for every small and sick newborn: key findings. Geneva: WHO; 2018.

6. World Health Organization (WHO). ICD-11: international statistical classification of diseases and related health problems. Geneva: WHO; 2018.

7. Leal MD, Esteves-Pereira AP, Nakamura-Pereira M, Torres JA, Theme-Filha M, Domingues RM, Dias MAB, Moreira ME, Gama SG. Prevalence and risk factors related to preterm birth in Brazil. Reprod Health 2016; 13(Supl. 3):127.

8. Platt MJ. Narrative Review: Outcomes in preterm infants. Public Health 2014; 128:399-403.

9. Barros FC, Rossello JLD, Matijasevich A, Dumith SC, Barros AJD, Santos IS, Mota D, Victora CG. Gestational age at birth and morbidity, mortality, and growth in the first 4 years of life: findings from three birth cohorts in Southern Brazil. BMC Pediatrics 2012; 12:169.

10. Moreira M, Lopes JMA, Carvalho MO. O recém-nascido de alto risco: teoria e prática do cuidar. Rio de Janeiro: Editora Fiocruz; 2004.

11. Bezerra FD, Menezes MAS, Mendes RB, Santos JMJ, Leite DCF, Kassar SB, Gurgel RQ. Cuidado perinatal em um estado do nordeste brasileiro: estrutura, processos de trabalho e avaliação dos componentes do essential newborn care. Revista Paulista de Pediatria 2019; 37(2):140-148.

12. Brasil. Ministério da Saúde (MS). Portaria No 930, de 10 de maio de 2012. Define as diretrizes e objetivos para a organização da atenção integral e humanizada ao recém-nascido grave ou potencialmente grave e os critérios de classificação e habilitação de leitos de Unidade Neonatal noâmbito do Sistema Único de Saúde (SUS). Diário Oficial da União 2012; 10 maio.

13. Brasil. Ministério da Saúde (MS). Atenção à saúde do recém-nascido: guia para os profissionais de saúde. Brasília: MS; 2014.

14. Brasil. Ministério da Saúde (MS). Portal da Saúde: SINASC. [site da Internet]. [acessado 2019 Nov 10]. Disponível em: http://portal.saude.gov.br/portal/ svs/ visualizar_texto.cfm?idtxt $=2132339$

15. Vilela MEA, Leal MC, Thomaz EBAF, Gomes MAM, Bittencourt S, Gama SGN, Silva L, Lamy Z. Avaliação da atenção ao parto e nascimento nas maternidades da Rede Cegonha: os caminhos metodológicos. Cien Saude Coletiva 2021; 26(3):789-800.
16. Pereira APE, Leal MC, Gama SGN, Domingues RMSM, Schilithz AOC, Bastos MH. Determining gestational age based on information from the Birth in Brazil study. Cad Saude Publica 2014; 30(Supl. 1):S59-S70.

17. Brasil. Ministério da Saúde (MS). Banco de dados do Sistema Único de Saúde-Datasus. [acessado 2020 Ago 10]. Disponível em http://www.datasus.gov.br

18. Torchin H, Ancel PY. Epidemiology and risk factors of preterm birth. J Gynecol Obstet Biol Reprod 2016; 45(10):1213-1230.

19. Pereira APE, Leal MDC, Gama SGN, Domingues RMSM, Schilithz AOC, Bastos MH. Determinação da idade gestacional com base em informações do estudo Nascer no Brasil. Cad Saude Publica 2014; 30(Supl. 1):S59-S70.

20. Costa MFS, Gomes Junior SC, Magluta C. Análise da distribuição dos nascimentos com marcadores de gravidade em maternidades com unidade de terapia intensiva neonatal no Sistema Único de Saúde. Cad Saúde Colet 2018; 26(2):125-130.

21. Ayebare E, Ntuyo P, Malande OO, Nalwadda G. Maternal, reproductive and obstetric factors associated with preterm births in Mulago Hospital, Kampala, Uganda: a case control study. Pan African Medical Journal 2018; 30:272

22. Brasil. Ministério da Saúde (MS). Portal da Saúde: SINASC. [site da Internet] [acessado 2020 Ago 10]. Disponível em: http://portal.saude.gov.br/portal/ svs/ visualizar_texto.cfm?idtxt $=2132339$

23. Gravena AAF, Paula MG, Marcon SS, Carvalho MDB, Pelloso SM. Idade materna e fatores associados a resultados perinatais. Acta Paulista de Enfermagem 2013; 26(2):130-135.

24. Nunes FBBF, Silva PC, Barbosa TLSM, Lopes MLH Silva EL. Influence of maternal age in perinatal conditions in live births of São Luís, Maranhão. Revista de Pesquisa: Cuidado é Fundamental Online 2020; 12:292-299.

25. Farias RV, Soares CFS, Araújo RS, Almeida VRS, Leitão DS, Santos JS, Santos LS, Nogueira SDA, Morais AC, Oliveira CBF. Gravidez na adolescência e o desfecho da prematuridade: uma revisão integrativa de literatura. Revista Eletrônica Acervo Saúde 2020; 56:3977.

26. Oliveira KA, Araújo EM, Oliveira KA, Cassoti CA, Silva CAL, Santos DB. Associação entre raça/cor da pele e parto prematuro: revisão sistemática com meta-análise. Rev Saude Publica 2018; 52:26.

27. Domingues RM, Dias MA, Schilithz AO, Leal MD Factors associated with maternal near miss in childbirth and the postpartum period: findings from the birth in Brazil National Survey, 2011-2012. Reprod Health 2016; 13(Supl. 3):115.

28. Pacagnella RC, Cecatti JG, Parpinelli MA, Sousa MH, Haddad SM, Costa ML, Brazilian Network for the Surveillance of Severe Maternal Morbidity study group. Delays in receiving obstetric care and poor maternal outcomes: results from a national multicentre cross-sectional study. BMC Pregnancy Childbirth 2014; 14:159. 
29. Lansky S, Lima Friche AA, Silva AA, Campos D, Azevedo Bittencourt SD, Carvalho ML, Frias PG, Cavalcante RS, Cunha AJ. Birth in Brazil survey: neonatal mortality, pregnancy and childbirth quality of care. Cad Saude Publica 2014; 30(Supl. 1):S1-15.

30. Leal MDC, Esteves-Pereira AP, Viellas EF, Domingues RMSM, Gama SGND. Prenatal care in the Brazilian public health services. Rev Saude Publica 2020; 54:08.

31. Menezes DCS, Leite IC, Ischramm JMA, Lela MDC. Avaliação da peregrinação anteparto numa amostra de puérperas no Município do Rio de Janeiro, Brasil, 1999/2001. Cad Saude Publica 2006; 22(3):553-559.

32. Menezes DCS. Caracterização dos riscos gestacionais e avaliação da adequação da internação hospitalar e peregrinação anteparto em uma amostra de puérperas no município do Rio de Janeiro 1999 - 2001 [dissertação]. Rio de Janeiro: Fiocruz; 2004.

33. Wildman K, Blondel B, Nijhuis J, Defoort P, Bakoula C. European indicators of health care during pregnancy, delivery and the postpartum period. Eur J Obstet Gynecol 2003; 111(Supl. 1):S53-S65.

34. Brasil. Ministério da Saúde (MS). Portaria no 1.459, 24 de junho de 2011. Institui, no âmbito do Sistema Único de Saúde, a Rede Cegonha. Diário Oficial da União 2011; 24 jun.

35. Bittencourt SDA, Gurgel RQ, Menezes MAS, Bstos LS, Leal MC. Neonatal care in Brazil: hospital structure and adequacy according to newborn obstetric risk. Paediatr Int Child Health 2015; 35(3):206-212.

36. Pilkington $\mathrm{H}$, Blondel B, Papiernik E, Cuttini M, Charreire H, Maier RF, Petrou S, Combier E, Künzel W, Bréart G, Zeitlin J. Distribution of maternity units and spatial access to specialised care for women delivering before 32 weeks of gestation in Europe. Health Place 2010; 16(3):531-538.
37. Viellas EF, Domingues RM, Dias MA, Gama SG, Theme Filha MM, Costa JV, Bastos MH, Leal MC. Prenatal care in Brazil. Cad Saude Publica 2014; 30(Supl. 1):S1-15.

38. Theme Filha MM, Ayers S, Gama SG, Leal M C. Factors associated with postpartum depressive symptomatology in Brazil: The Birth in Brazil National Research Study, 2011/2012. J Affect Disord 2016; 194:159-167.

39. Zeitlin J, Gwanfogbe CD, Delmas D, Pilkington H, Jarreau PH, Chabernaud JL, Bréart G, Papiernik E. Risk factors for not delivering in a level III unit before 32 weeks of gestation: results from a population-based study in Paris and surrounding districts in 2003. Paediatr Perinat Epidemiol 22(2):126-135.

Artigo apresentado em 06/06/2020

Aprovado em 27/10/2020

Versão final apresentada em 29/10/2020

Editores-chefes: Romeu Gomes, Antônio Augusto Moura da Silva 